\title{
»Du wirst essen und nicht satt werden...» Die Angst vor dem Hunger ${ }^{1}$
}

Sara Kipfer

Hunger ist vermutlich das kontinuierlichste Problem, mit dem sich Menschen durch alle Zeiten hindurch auseinandersetzen mussten. Entsprechend zählt »Hungersnot» zu den mächtigsten, durchdringendsten und (wohl) emotionalsten Wörtern des historischen Vokabulars. ${ }^{2}$ Auch in der Hebräischen Bibel ist das Thema stark emotional aufgeladen. Dies macht es schwierig, wenn nicht sogar unmöglich, überhaupt zu definieren, was in den Texten mit »Hunger" beziehungsweise "Hungersnot" gemeint ist. Wie lange muss eine Trockenheit andauern, bis sie als Bedrohung wahrgenommen wird? Welche Rolle spielen nicht nur die Niederschlagsmenge (sog. meteorologische Dürre), sondern auch der Zeitpunkt des Niederschlags (sog. landwirtschaftliche Dürre), eine kriegerische Zerstörung, soziale Ungerechtigkeit, Probleme in Zusammenhang mit der Vorratsbildung, Heuschreckenschwärme, Überschwemmungen oder Getreidekrankheiten? Wie - wenn überhaupt - schlägt sich die Erfahrung des Hungers in den biblischen Texten nieder?

Es ist nicht nur diese Multikausalität, die zu unterschiedlichen Katastrophenwahrnehmungen führt und die Thematisierung von historischen Hungersnöten in den biblischen Texten erschwert, sondern entscheidend ist auch, dass Hungersnöte - anders als Erdbeben

\footnotetext{
1 Diese Publikation ist Teil meines Habilitationsprojektes und wird gefördert durch den Europäischen Sozialfonds sowie durch das Ministerium für Wissenschaft, Forschung und Kunst Baden-Württemberg.Vgl. die wesentlich ausführlichere englische Publikation: Sara Kipfer, "You ate, but you never have enough...«: Fear of Famine and Food Shortage in the Hebrew Bible and the Ancient Near East, in: Welt des Orients (im Druck).

2 See David Arnold, Famine. Social Crisis and Historical Change, Oxford 1988, 5 : "Famine is one of the most powerful, pervasive, and arguably one of the most emotive words in our historical vocabulary and that in itself makes it all more difficult to isolate its meaning and wider significance."

3 Luzia Sutter-Rehmann, Wut im Bauch. Hunger im Neuen Testament, Gütersloh 2014, betont die Schwierigkeit aus den neutestamentlichen Texten etwas über Hungersnöte zu erfahren und entwirft eine Hermeneutik, "die Hinweise auf Hunger aufspürt und Verbindungen sichtbar macht, auch wenn im Text nicht explizit von Hunger geredet wird « (ebd., 15). Trotz der vier von ihr definierten text marker - Reden über das Essen, Schweigen über den Hunger, emotionale Unruhe (dazu zählt insbesondere die Wut) und Verweise auf die Tora (ebd., 109) - bleibt dieser Textzugang spekulativ.
} 
oder Überschwemmungen - auch schleichend auftreten können und nicht selten in Kombination mit anderen Katastrophen - etwa kriegerischer Zerstörung - erfahren werden. Aus diesem Grund sind die Risikoeinschätzungen sehr vielfältig. Mit der diffusen Gefährdungswahrnehmung geht die Angst vor einer unkontrollierbaren Bedrohung einher.

Für die meisten Menschen in Europa ist Hunger eine weit entfernte Katastrophe, die in ihren Bildern und Resonanzen einer vergangenen Welt angehört. ${ }^{4}$ Erst in den vergangenen Jahren hat sich dieses Bewusstsein wieder geändert. Mit den sich anbahnenden oder tatsächlich eingetretenen Handelskriegen zwischen den USA und anderen Ländern der Welt und mit der durch die Dürren (2003, 2010, 2013, 2015 und insbesondere 2018) in Europa "wahrnehmbar" gewordene Klimaveränderung, wurde deutlich, dass Ernährungssicherheit auch in Europa keine Selbstverständlichkeit ist. Der anthropogen verursachte Klimawandel prägt - spätestens seit dem Hitzesommer 2018 in Europa und der Gründung der \#FridaysForFuture im August des gleichen Jahres - den gesellschaftlichen und politischen Diskurs. Geäussert werden nicht nur politische Forderungen und konkrete Massnahmen, sondern es macht sich auch eine diffuse Angst in der Bevölkerung breit. ${ }^{5}$ In zahlreichen Ländern wird mittlerweile der Klimawandel als grösste Gefahr angesehen - gefolgt von islamistischen Terrorgruppen und Cyber-Attacken. ${ }^{6}$ Die Angst vor extremen Klimaereignissen und einer globalen Klimaveränderung ist in der breiten Bevölkerung angekommen, so dass gelegentlich sogar von "Klimatrauma" mit den Symptomen einer prätraumatischen Belastungsstörung die Rede ist. ${ }^{7}$ Angst war und ist ein wichtiger

4 Vgl. ausfuhrlich dazu Brian Murton, Art.VI.4 Famine, in: The Cambridge World History of Food, Vol. 2, hg. v. Kenneth F. Kiple/Kriemhild Coneè Ornelas, Cambridge/New York et al. 2000, 1411-1427; Evan D. G. Fraser/Andrew Rimas, Empires of Food. Feast, Famine, and the Rise and Fall of Civilizations, Berkeley 2010.

5 Arnold, Famine, 1: "Although for most people in the present-day western world famine is a distant hunger, more Old Testament than contemporary in its images and resonances, the prospect of famine on a massive, possibly global, scale still haunts our collective fears and imagination."

${ }_{6}$ Pew Research Center, Jacob Poushter/Christine Huang, Climate Change Still Seen as the Top Global Threat, but Cyberattacks a Rising Concern, https://www. pewresearch.org/global/2019/02/10/climate-change-still-seen-as-the-top-globalthreat-but-cyberattacks-a-rising-concern/ (16.12.2019).

7 Vgl. ausführlich zum Begriff »Klimatrauma« und seinen unterschiedlichen Anwendungen: Sara Kipfer, Klimakatastrophen wahrnehmen, deuten und bewältigen. Jer $14^{*}$ und Joel 1-2* als Klage anlässlich einer Dürre, in: Alttestamentliche Exegese im Lichte der Traumaforschung, hg. v. Dorothea Erbele-Küster/Nikolett Móricz/Manfred Oeming, (im peer review). 
politischer, sozialer, religiöser und gesellschaftlicher Motor $^{8}$ und es lohnt sich daher die Muster mit denen in der Hebräischen Bibel, die Angst vor dem Hunger geschürt, beziehungsweise bewältigt wurde, $\mathrm{zu}$ analysieren und nach Ähnlichkeiten und Unterschieden im gegenwärtigen gesellschaftlichen Diskurs zu fragen. ${ }^{9}$

In den Prophetenbüchern ist die Angst vor extremen Klimaereignissen, insbesondere dem ausbleibendem Niederschlag und damit zusammenhängenden Hungersnöten, allgegenwärtig - wenn auch lediglich selten explizit. ${ }^{10}$ Zwar werden Hungersnöte in den Prophetenbüchern gelegentlich als aktuelle Krisen und alltägliche Erfahrung beschrieben, viel häufiger findet jedoch die Hungersnot als künftige drohende Katastrophe Erwähnung. ${ }^{11}$ Aber auch die Gefährdungswahrnehmung sagt etwas aus über die tatsächlichen historischen Ereignisse - allerdings ist sie stark kontextuell geprägt und nicht so einfach zu verstehen und einzuordnen. Unter dieser Prämisse sollen die Texte im Folgenden - bei aller Vorsicht - auf besondere Bedingungen und Erfahrungskonstellationen hin analysiert und als Gestaltwerdung von Lebensvorgängen und sprachlicher Bewältigung gelebten Lebens betrachtet werden. ${ }^{12}$

Die Kommunikationsstruktur in den Texten ist dabei von entscheidender Bedeutung: Mit dem Hunger wird in den Prophetenbüchern immer wieder gedroht. Umgekehrt wird aber auf die

\footnotetext{
8 Vgl. Martha C. Nussbaum, The Monarchy of Fear. A Philosopher Looks at Our Political Crisis, New York 2018.

9 Selbstverständlich kann dies in dem beschränkten Rahmen lediglich sehr skizzenhaft geschehen.

${ }_{10}$ Ausnahmen sind etwa Ez 4,16 und 12,18f., wo gesagt wird, dass Menschen in

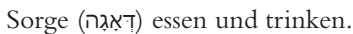

11 Ähnlich hat Seth Richardson, Obedient Bellies: Hunger and Food Security in Ancient Mesopotamia, in: Journal of the Economic and Social History of the Orient 59 (2016), 750-792, hier 770, dies auch für mesopotamische Texte festgehalten: "In sum, expressions describing food shortages in omens, curses, literary-historical, and administrative texts in particular contexts cannot be depended on as descriptive of actual crises and everyday realities." Und er führt aus: „Even as one concedes that many euphemic and idiomatic references to hunger are not documentary, the infusion of the topic into poetic and administrative language shows that the motif of hunger gave conceptual shape to social hopes and fears, continuously animating the contingent disasters which were the raison d'être for administration itself."

${ }^{12}$ So die Forderung des früheren Zürcher Alttestamentlers Odil Hannes Steck, Welt und Umwelt, Stuttgart u.a. 1978, 45: „Sie [die biblisch-historische Exegese] darf sich nicht auf Analyse und Deskription erklärungsbedürftiger Sätze beschränken, sondern muß biblische Texte historisch in ihr dynamisches Lebensumfeld mit besonderen Bedingungen und Erfahrungskonstellationen betten und sie als Gestaltwerdung von Lebensvorgängen und als sprachliche Bewältigung gelebten Lebens sehen.»
} 
kollektive Angst ${ }^{13}$ und den existierenden Hunger reagiert und eine wunderbare Zukunft jenseits der harten Realität versprochen. Im Folgenden stehen Texte im Vordergrund, die eine Hungersnot als ein mögliches künftiges Ereignis im Blick haben und daher auch als mögliche Bedrohung und Auslöser von Angst verstanden werden können, beziehungsweise die die Vermittlung von Trost und Hoffnung zum Gegenstand haben. Es geht dabei in erster Linie um die Frage, was für Ängste und Befürchtungen konkret geschürt werden und welche Bewältigungsstrategien umgekehrt die Texte im Umgang mit Angst aufzeigen. In einem ersten Schritt werden einerseits Gerichtsworte und Anklagen als Teil der Unheilsprophetie (auch als Schelt- und Drohwort bekannt), zum anderen sogenannte "Vertragsflüche«, die sich sowohl in der Hebräischen Bibel als auch in aramäischen Texten (9./8. Jh. v.Chr.) finden, näher beleuchtet. In einem zweiten Schritt werden anschließend Beispiele aus der Heilsprophetie präsentiert, die genau diese Formulierungen aufnehmen und aus den Drohungen, Heilszusagen machen. Abschließend sollen das Verhältnis von Dystopie und Utopie ${ }^{14}$ und der entsprechende Umgang mit der Angst vor dem Hunger näher beleuchtet werden.

\footnotetext{
${ }^{13}$ Vgl. zur kollektiven Angst Elisabeth Wagner-Durand, Beyond texts? Potentials and Limitations of a Holistic Approach to Collective Fear in Mesopotamia/ANE, in: WdO (im Druck).Vgl. zur Emotion "Angst« in der Hebräischen Bibel generell Sara Kipfer, Angst, Furcht und Schrecken. Eine kognitiv-linguistische Untersuchung einer Emotion im Biblischen Hebräisch, in: JNSL 42 (2016), 15-79 und Sara Kipfer, Art. "Furcht (Erschrecken, Ehrfurcht, Gottesfurcht / Gottesfurchtige)«, in: Wibilex, hg. v. Michaela Bauks/ Klaus Koenen/Stefan Alkier, 2017, http://www.bibelwissenschaft. de/de/stichwort/18774/ (10.5.2020).

${ }^{14}$ Vgl. Ágnes Heller, Von der Utopie zur Dystopie. Was können wir uns wünschen?, Wien/Hamburg 2016, 17: „Utopien sind Schöpfungen der Einbildungskraft verbunden mit bestimmten Ansichten einer Zeit und der Leidenschaft der Hoffnung. Dystopien sind Schöpfungen der Einbildungskraft in Verbindung mit bestimmten Ansichten der Zeit und der Leidenschaft der Furcht. Da alle gesunden Menschen überall und immer Hoffnung und Furcht empfinden, hängt der Unterschied zwischen Utopien und Dystopien von den herrschenden Ansichten ab.«
} 


\section{Die Angst vor dem Hunger in der Unheilspro- phetie und in Vertragstexten}

In der Unheilsprophetie und in Vertragstexten werden ähnliche Formulierungen verwendet. Beide greifen sie auf sogenannte "Nichtigkeitsflüche" (englisch: "futility curses») zurück. ${ }^{15}$ Diese "Nichtigkeitsflüche "zielen darauf ab, menschliche Handlungen als vergeblich zu charakterisieren. Sie bestehen jeweils aus einem ersten Satz (Protasis), der eine Handlung beschreibt, und werden gefolgt von einem zweiten Satz (Apodosis), in der die Handlung verneint beziehungsweise deren Unwirksamkeit betont wird (durchgängig mit "aber nicht" ולא eingeleitet). Ferner kann die Vergeblichkeit auch durch die Struktur maximalen Aufwand furr minimalen Gewinn (ebenfalls mit "aber nicht « ולא eingeleitet) thematisiert werden. ${ }^{16}$ Während im Fall der Unheilsprophetie der Hunger als unweigerliche Konsequenz vorhergesagt wird, wird in den Vertragstexten eine Hungersnot lediglich im Falle eines Vertragsbruchs als mögliches künftiges Ereignis angedroht und es fehlt das anklagende Element. ${ }^{17}$ Einer der charakteristischen Texte, der auf so einen "Nichtigkeitsfluch" zurückgreift, ist Micha 6,9-15. ${ }^{18}$

Micha 6,9-15

V. 9 Jhwhs Stimme ruft der Stadt:

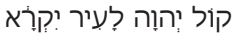

Klugheit ist es, deinen Namen zu fürchten [MT »sehen«]

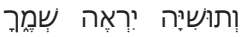

Hört, Stamm und "Stadtversammlung».

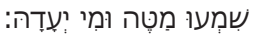

\footnotetext{
${ }^{15}$ Der Terminus geht auf Delbert R. Hillers, Treaty-curses and the Old Testament Prophets, Rome 1964, 28, zurück. Die Charakterisierung als "Fluch« trifft nur auf einen Bruchteil der Texte formal tatsächlich zu, weshalb der Fachterminus nicht unproblematisch ist.

${ }^{16}$ Vgl. William S. Morrow, Famine as the Curse of Kings: Royal Ideology in Old Aramaic Futility Curse Series, in: Herrschaftslegitimation in vorderorientalischen Reichen der Eisenzeit, hg. v. Christoph Levin/Reinhard Müller, Tübingen 2017, 111-124.

${ }^{17}$ Jörg Jeremias, Kultprophetie und Gerichtsverkündigung in der späten Königszeit Israels, Neukirchen-Vluyn 1970, 165, unterscheidet zwischen "prophetischer Gerichtsankündigung gegen Israel" und "kultischer Fluchverkündigung». Diese Unterscheidung ist in der jüngsten Forschung aufgegeben worden.Vgl. beispielsweise Laura Quick, Deuteronomy 28 and the Aramaic Curse Tradition, Oxford/New York 2018.

${ }^{18}$ Vgl. Hans Walter Wolff, Micha, Neukirchen-Vluyn 1982, 161; Jörg Jeremias, Die Propheten Joel, Obadja, Jona, Micha, Göttingen 2007, 206f.; Rainer Kessler, Micha, Freiburg i.Br./Basel/Wien 1999, 275f.
} 
V.10 Kann ich auf Dauer vergessen das unrechte Bat (Flüssigmass),

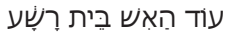

die Schätze des Unrechts

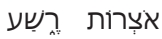

und das geschrumpfte, verwünschte Epha (Trockenmass)

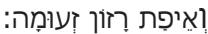

V. 11 Stehe ich rein da bei unrechter Waage

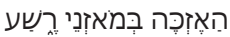
und bei einem Beutel mit falschen Gewichtssteinen?

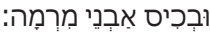

V. 12 Weil ihre [Jerusalems oder Samarias] Reichen voll sind von Gewalttat

und ihre Bewohner Lügen reden

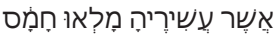

und ihre Zunge Betrug ist in ihrem Mund,

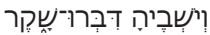

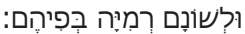

V. 13 doch ich, ich mache wund, indem ich dich schlage,

indem ich veröden lasse wegen deiner Sünden.

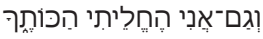

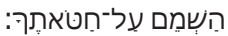

V. $14 \mathrm{Du}$ wirst essen,

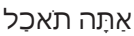

aber nicht satt werden.

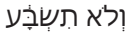

Dein Hungergefühl in deiner Mitte.

Und du wirst einholen,

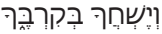

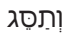

aber nicht in Sicherheit bringen.

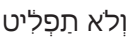

Und was du in Sicherheit bringst, übergebe ich dem Schwert.

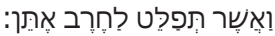

V. 15 Du wirst säen,

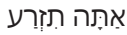

aber nicht ernten.

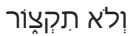

Du wirst Oliven keltern,

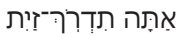

aber dich nicht mit Öl salben.

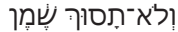

Und Trauben (wörtl. »Most«) [keltern],

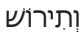
aber keinen Wein trinken.

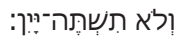

Im Fall von Micha 6,14-15 ist eindeutig, dass die Strafe erst künftig eintreten wird. Gott lässt die Menschen der Stadt "wegen ihrer Sün-

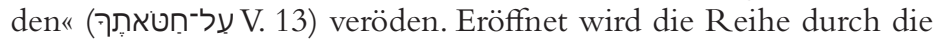
Drohung "du wirst essen, aber nicht satt werden... « sowie dem noch verstärkenden und ergänzenden Zusatz, dass das Hungergefühl ${ }^{19}$ "in deiner Mitte« bleiben wird. Es geht also um nicht mehr und nicht weniger als um die Ankündigung einer bevorstehenden Hungersnot. Wodurch diese verursacht wird, wird jedoch nicht näher erläutert. Im zweiten "Nichtigkeitsfluch" wird gesagt, dass die Menschen zwar einholen, aber nicht in Sicherheit bringen, weshalb vermutet wurde,

${ }^{19}$ So übersetzt Jeremias, Die Propheten Joel, Obadja, Jona, Micha, 206. Textkritisch

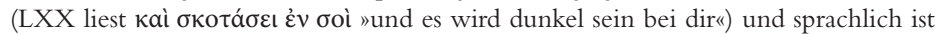
der Ausdruck ?שֶׁ? (Hapaxlegomenon) unsicher. Kessler, Micha, 275, übersetzt mit "Kot». 
dass hier auf die Vergeblichkeit Nahrungsmittelvorräte anzulegen, angespielt werde. ${ }^{20}$ Es folgen drei »Nichtigkeitsflüche«, bei denen der agrarische Kontext im Mittelpunkt steht: säen und nicht ernten, Oliven keltern und sich nicht salben, Trauben (wörtl. »Most«) pressen und keinen Wein trinken. Die konkrete Ursache bleibt jedoch merkwürdig offen. Während »säen und nicht ernten« an eine Dürre denken lassen könnte, legt sich für die beiden abschliessenden "Nichtigkeitsflüche" eher ein kriegerischer Kontext nahe, denn offenbar werden die Felderträge (Oliven und Trauben) ja geerntet und weiter verarbeitet, verhindert wird lediglich deren Konsum. Der ganz konkreten Kritik - nämlich dem wirtschaftlichen Betrug, dem ungerechten Handel (gefälschte Hohlmasse und Gewichte) - steht also eine sehr diffuse Gerichtsankündigung gegenüber, deren Ursachen alles andere als eindeutig sind.

In Am 5,7.10-12 wird der Zusammenhang zwischen der Kritik an der gegenwärtigen Situation und dem zukünftig eintretenden Gericht explizit begründet: Weil die Gerichtsbarkeit missachtet wird (V. 10), Pachtzins und Steuern eingetrieben werden (V. 11), deshalb (לְרן) wird die Ordnung aufgehoben und der Zusammenhang von Häuser bauen und darin wohnen, Weingärten pflanzen und Wein trinken, zerbrochen. Auch hier bleibt die Drohung höchst unkonkret; es ist kein Feind, der hier im Blick ist, es wird kein konkretes Zukunftsszenario ausgemalt, sondern lediglich festgehalten, dass das, was einmal war, was gebaut und gepflanzt wurde, keine Selbstverständlichkeit für alle Zeiten hat.

Am 5,7.11

V. 7 Ihr, die ihr Recht in Wermut verkehrt und die Gerechtigkeit zu Boden stosst:

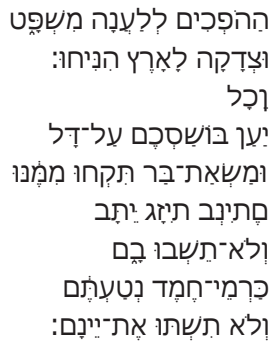

Die Strafe trifft nicht alle, sondern in erster Linie die Oberschicht, die das Recht und die Gerechtigkeit missachtet und Pachtzins und

\footnotetext{
${ }^{20}$ Vgl. beispielsweise Quick, Deuteronomy 28, 123. Diese Interpretation ist möglich, jedoch auf Grund der unsicheren Bedeutung der Wörter סוג (Hifil) und פלט (Hifil) nicht sicher belegt.
} 
Getreideabgaben erhebt. Dass die Strafe offensichtlich wirklich nur jene trifft, die falsch gehandelt haben und nicht das ganzeVolk, wird noch deutlicher in Hos 4,4-12. Hier stehen die Priester im Fokus der Anklage. Weil sie Gott verlassen und an Hurerei und Wein festgehalten haben, soll auch ihre künftige Handlung derVergeblichkeit preisgegeben werden (das Subjekt in V. 10 ist allerdings unsicher).

Hos 4,9-11

V. 9 Und wie das Volk, so der Priester:

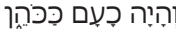

Seiner Wege wegen werde ich ihn heimsuchen,

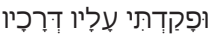
und seine Taten werde ich ihm vergelten.

V. 10 Und sie werden essen,

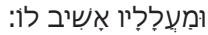
und nicht satt werden;

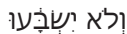
Hurerei treiben,

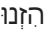
und sich nicht ausbreiten;

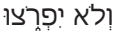

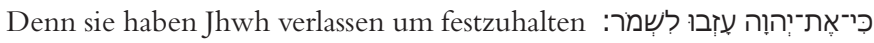
V. 11 an Hurerei und Wein und Most

Sie rauben das Herz!

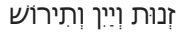

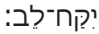

Auffallend wird hier nicht jemand in der 2. Person angesprochen (weder im Sg. wie in Micha 7,14f. noch im Pl. wie in Am 5,11), sondern es wird über das Volk beziehungsweise die Priester gesprochen. Eine ähnliche Situation findet sich auch in Zef 1,12-13. Diese Unheilsprophetie richtet sich gegen Männer, »die auf ihren Weinhefen eindicken " und Gottes Handeln in Frage stellen. Diese Reichen sollen enteignet werden, ihr Besitz geplündert und ihre Häuser der Verwüstung anheimfallen. Obwohl sie Häuser bauen, werden sie nicht mehr darin wohnen und obwohl sie Weinberge pflanzen, keinen Wein mehr trinken. Auch ihre Handlungen werden vergeblich.

Zef 1,12-13

V. 12 Und geschehen wird es in jener Zeit:

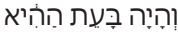

Ich durchsuche Jerusalem mit Leuchten

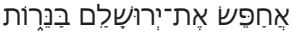
und werde einschreiten gegen die Männer,

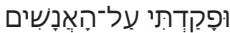
die auf ihren Weinhefen eindicken, die in ihrem Herzen sagen:

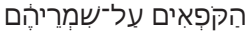

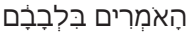

»Weder Gutes wirkt Jhwh

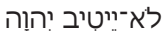

noch Böses."

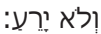

V. 13 Dann verfällt ihr Besitz der Plünderung und ihre Häuser derVerwüstung.

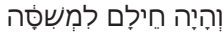

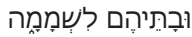

Und sie bauen Häuser

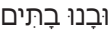

und werden nicht darin wohnen,

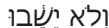


und sie pflanzen Weinberge,

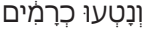

aber werden nicht deren Wein trinken.

Sprecher und Angesprochene sind nicht stereotyp, sondern können ausgetauscht werden (direkte Jhwh-Rede z.B. Mi 6,9-16; Zef 1,1213, beziehungsweise prophetisches Gerichtswort Hos 4,10b-11); dennoch scheinen sämtliche Texte auf einen ähnlichen soziologischen, politischen und historischen Kontext zurück zu führen: Kritisiert wird eine Oberschicht - Händler (so Mi 6,10f.), Grossgrundbesitzer (so lässt sich aus Am 5,11 schliessen), Priester (Hos 4,9), beziehungsweise die "Wohllebenden" (Zef 1,12). Ihr Vergehen ist

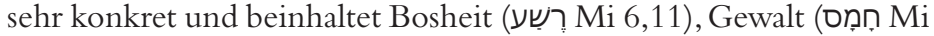

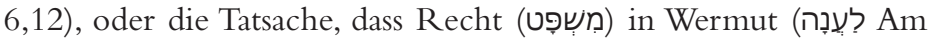
5,7) verkehrt und Gerechtigkeit (צִדָקָה zu Boden gebracht wird (Am 5,7). Konkreter werden Hurerei (זָנוּ Hos 4,11), der exzessive Weingenuss (Hos 4,11; Zef 1,12), die Anhäufung von Wohlstand und Landbesitz (Am 5,11) und Betrug (gefälschte Waage und betrügerische Gewichtssteine Mi 6,11) kritisiert. Religiöse Kritik wie das Verlassen Gottes (Hos 4,10), die fehlende Gotteserkenntnis (דָ דִעת Hos 4,6) und Sünde gegen Gott generell (חטא Hos 4,7) beziehungsweise die Unterstellung, dass Gott "unwirksam» sei (Zeph 1,12; vgl. Jes 5,12), spielen ebenfalls eine wichtige Rolle.

Das Gericht wird eintreten "in jener Zeit" (בָעַת הַהִיא Zef 1,12). Wodurch es genau verursacht wird, das bleibt jedoch meist offen. Entscheidend ist, dass sich Schuld und Strafe in jedem Fall entsprechen:Wer masslos konsumiert hat, wird nicht mehr satt werden; wer sich unrechtmässig genommen hat, wird dafür bestraft werden. Was als Gericht für die einen erscheint, mag als Hoffnung für die anderen aufleuchten. Die Botschaft der Propheten ist eindeutig: Das gegenwärtige Handeln bleibt nicht folgenlos, sondern hat Konsequenzen für die Zukunft.

Was ist jedoch mit »essen und nicht satt werden" genau gemeint? Bedeutet es, dass tatsächlich auf Grund von Dürre oder Krieg nicht genug Nahrungsmittel vorhanden sind, oder soll damit zum Ausdruck gebracht werden, dass eine Handlung - also beispielsweise "essen" - vergeblich ist und nicht zu einem Sättigungsgefühl führen wird?21

\footnotetext{
${ }^{21} \mathrm{Vgl}$. Thomas Podella, Notzeit-Mythologien und Nichtigkeitsfluch, in: Religionsgeschichtliche Beziehungen zwischen Kleinasien, Nordsyrien und dem Alten Testament, hg. v. Bernd Janowski/Klaus Koch/Genot Wilhelm, Freiburg, Schweiz/Göttingen, 1993, 427-454, hier 444.
} 
Für die Annahme, dass hier eine tatsächliche Hungersnot angekündigt wird, spricht, dass die Menschen in der Antike tatsächlich an die Wirksamkeit dieser Flüche geglaubt haben. Es ist sogar ein konkreter Fall von Hungersnot bekannt, bei der ein sog. Nichtigkeitsfluch als "Deutungsmuster" diente. Ein Bericht des assyrischen Königs Assurbanipals (669-629 v.Chr.) beschreibt, was einem arabischen Stamm widerfahren ist und erklärt dies mit einem Vertragsbruch. ${ }^{22}$

\section{Rassam Zylinder Col IX 58-74 (BM 91026)23}

Mangel brach unter ihnen aus. [...] Die Kameljungen, die jungen Esel (?), die Wildkälber saugten siebenmal an säugenden (Tieren) und konnten ihren Bauch mit Milch nicht sättigen. Die Einwohner von Aribi frugen einer den anderen, gegenseitig: "Warum ist dem Lande Arubu (Aribi) eine derartig schlimme Sache widerfahren?" (und antworteten sich darauf selbst) also: "Weil wir die großen Verträge Ašsurs nicht beobachtet, (weil) wir uns versündigt haben gegen die Wohltat Assurbanipals, des Königs, den Ellil liebt. [...].«

In den assyrischen Verträgen treten verschiedene Flüche auf (z.B. einfache Flüche mit oder ohne Anrufung einer Gottheit und Analogieflüche). Es sind daneben auch aramäische Verträge bekannt, die "Nichtigkeitsflüche" enthalten. So findet sich etwa folgende Drohung auf der Statue von Tell Feherīye aus dem 9. Jahrhundert: ${ }^{24}$

Statue von Tell Feherīye, Z. 18-22 (KAI $\left.{ }^{5} 309\right)^{25}$

Und er möge (19) säen, aber nicht ernten! זיע

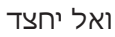
Und möge er tausend $[\mathrm{Ma} \beta]$ Gerste säen,

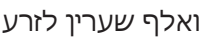

\footnotetext{
${ }^{22}$ William S. Morrow, Famine as the Curse of Kings: Royal Ideology in Old Aramaic Futility Curse Series, in: Herrschaftslegitimation in vorderorientalischen Reichen der Eisenzeit, hg. v. Christoph Levin/Reinhard Müller, Tübingen 2017, 111-124, hier 114 , meint, dass es kaum Zweifel daran geben kann "that Assurbanipal's annals recall the wording of a specific treaty made between Assyria and its Arab subjects".

${ }^{23}$ Die Übersetzung stammt von Maximilian Streck, Assurbanipal und die letzten assyrischen Könige bis zum Untergang Niniveh's, Teil II:Texte, Leipzig 1916.

${ }^{24}$ Ganz ähnliche Formulierungen finden sich auch auf der Sfire Stele 1, A 20*-24* $\left(\mathrm{KAI}^{5} 222\right)$ und Sfire Stele 2, A 1-3 (KAI $\left.{ }^{5} 223\right)$ sowie auf der Bukān Stele, Zeile 5-10 (KAI ${ }^{5}$ 320) (8. Jh.).

${ }^{25}$ Der Text ist Dirk Schwiderski, Die alt- und reichsaramäischen Inschriften, Bd. 1: Konkordanz, Berlin/New York 2008, 194 entnommen. Die deutsche Übersetzung ist in Anlehnung an Wilhelmus C. Delsman, Aramäische historische Inschriften, TUAT, Bd. 1, 637, verfasst.
} 
aber nur ein parîs davon einnehmen!

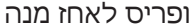

(20) Und mögen hundert Mutterschafe ein Lamm säugen,

aber es wird nicht gesättigt!

(20) ומאה סאון להינקן אמר

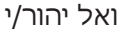

Und mögen hundert Kühe (21) ein Kalb säugen,

aber es wird nicht gesättigt!

ומאה סור להינקן (21) עגל

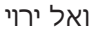

Die Flüche haben unterschiedliche Funktionen, je nach dem, ob sie eine Inschrift oder einen Vertrag als solchen schützen wollen. ${ }^{26}$ Sie nennen allesamt Gottheiten als Garanten, die in Aktion treten, wenn gegen die Inschrift verstossen oder derVertrag gebrochen wird. Die Flüche in diesen Verträgen haben die Funktion, den staatlichen, sakralen, justiziellen und sozialen Bereich zu schützen. ${ }^{27}$ In Lev 26,14-15 erfolgt eine Bestrafung durch die Flüche dann, wenn das Volk Gott nicht gehorcht und die Gebote nicht hält und dadurch den Bund bricht. Auch in diesem Fall wird neben zahlreichen weiteren Strafen mit "Nichtigkeitsflüchen" gedroht. In Dtn 28,15$44^{*}$ ist der Einzelne für seine Taten verantwortlich: er wird bestraft, wenn er nicht alle Gebote und Verordnungen Gottes beachtet. Auf die Verheißung von Segen in Dtn 28,7-14 folgen unterschiedliche Fluchankündigungen. Während die Flüche in Dtn 28,30-33 eine kriegerische Situation voraussetzen und beispielsweise die Situation in den Blick rückt, dass ein Mann zwar mit einer Frau verheiratet ist, aber ein anderer Mann mit ihr schlafen wird und dass er seines Viehbesitzes enteignet wird, setzen jene in Dtn 28,38-40 erneut eine agrarische Notsituation voraus: Die Heuschrecke frisst die Saat ab, die Weinberge werden von Würmern heimgesucht, und die Oliven fallen vor der Erntezeit ab.

In einigen Texten scheint allerdings nicht der tatsächliche Mangel an Nahrung im Vordergrund zu stehen; vielmehr lässt sich der Hunger auf Grund der Vergeblichkeit der Nahrungseinnahme nicht stillen. DieVergeblichkeit einer Handlung betrifft auch andere Bereiche und findet sich etwa in der Opposition von ssuchen und nicht fin-

\footnotetext{
${ }^{26}$ Vgl. Krzysztof J. Baranowski, The Old Aramaic and Biblical Curses, in: Liber Annuus 62 (2012), 173-201, hier 198. Melissa Ramos, A Northwest Semitic Curse Formula: The Sefire Treaty and Deuteronomy 28, in: ZAW 128 (2016), 205-220, hier 219 , kommt zum Schluss, dass "the spread of imprecations, in particular, suggests a purposeful use of ritual oath practice intended to instill fear and promote the stability of the Empire at its distant borders."

${ }^{27}$ Vgl. Walter Sommerfeld, Umweltzerstörung und ökologische Krisen im Alten Orient, in: State Formation and State Decline in the Near and Middle East, hg. v. Rainer Kessler/Walter Sommerfeld/Leslie Tramontini, Wiesbaden 2016, 15-49.
} 
den « in Jes 31,12; Hos 2,9a; 5,6; Am 8,12b und Spr 1,28b, die sämtlich der Syntax der "Nichtigkeitsflüche» folgen (durchgehend mit ולא ${ }^{28}$ Auch im Bereich der Sexualität ist davon die Rede, dass man zwar Hurerei treiben, aber dabei nicht "befriedigt" (wörtlich "satt" שבעy) wird (Ez 16,28-29). Diese Beispiele lassen vermuten, dass in einigen Fällen möglicherweise nicht der Konsum von Nahrungsmitteln allein ein Problem darstellt, sondern vielmehr die Möglichkeit, von dem was man isst, satt zu werden. In diesen Fällen geht die Erwartung an die Zukunft über die Erfahrung der Vergangenheit hinaus. Stattdessen produzieren die Texte dystopische Vorstellungen von der Unmöglichkeit einer Bedürfnisbefriedigung. Es geht nicht um Nahrungsmittelknappheit oder um Nahrungsmittelverteilung an sich, sondern um die grundsätzliche Unmöglichkeit, "satt zu werden «, ja um die Nutz- und Sinnlosigkeit menschlicher Handlung insgesamt (vgl. Jer 12,13a "sie haben sich abgemüht und hatten kei-

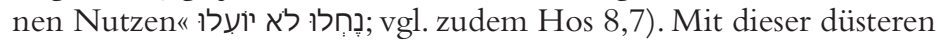
Zukunftsprognose, die schlimmer ist als das, was je erlebt wurde, wird also eindeutig "Angst" gemacht. Diese Angst vor der Zukunft spielt eine zentrale Rolle in der Textpragmatik dieser Beispiele.

\section{Die Hoffnung auf eine Zukunft ohne Hunger in der Heilsprophetie}

Die Unheilsprophetie hatte also zum Ziel, eine gerechte Strafe für das ungerechte Verhalten einzelner Bevölkerungsgruppen anzudrohen. Sie verwendete dabei Formulierungen, wie sie aus aramäischen und neuassyrischen Verträgen bekannt sind, und spitzte diese im Laufe der Zeit immer mehr zu. Sie machte schließlich Angst vor etwas, das über die menschliche Erfahrung hinaus geht. Wie lange diese Unheilsankündigung dauerte, wie viele Generationen von ihr erfuhren, ist schwer zu sagen. Fest steht, dass sie nicht das Werk eines einzelnen Redaktors war, denn zu unterschiedlich sind die Formulierungen, und zu konkret ist, was jeweils kritisiert wird. Sie dokumentieren sehr explizit, wie einzelnen Bevölkerungsgruppen für ihr Verhalten Unheil angedroht und Angst gemacht wurde.

In späterer Zeit wurden diese Dystopien jedoch in Utopien umgewandelt, aus der Unheilsprophetie wurde Heilsprophetie. Jer

${ }^{28}$ In einigen Stellen ist auch davon die Rede, dass das Volk Jhwh anruft, er ihnen aber nicht antwortet (vgl. Mi 3,4a; Sach 7,13; Jer 11,11b). Diese Stellen sollten nicht zu den "Nichtigkeitsflüchen" gerechnet werden, da das Subjekt wechselt (vgl. auch Mal $1,4 b)$. 
31,4f.; Ez 28,26 und Am 9,13-15 beinhalten alle unterschiedliche Verheissungen, dass die Menschen nach dem Krieg ihre Städte wieder aufbauen, vor allem aber, dass sie wieder Gärten und Weinberge bepflanzen werden. ${ }^{29}$ In Aussicht gestellt wird eine wunderbare Zukunft, in der die Flüche mehr oder weniger explizit ihre Kraft verlieren (Jes 62,8-9) und das Gericht aufhört (Am 9,14).

Ein frühes Beispiel für so eine Verheißung findet sich vermutlich in Dtn 6,10-12 (vgl. auch Dtn 8,11-18*). Hier wird an das Versprechen, dass Gott Israel ein Land zugesagt, in dem sie leben können, ohne dafür gearbeitet zu haben, erinnert. Es handelt sich also um eine Umkehrung dessen, was zuvor in der Unheilsprophetie angedroht wurde. Gelegentlich wurde diese Umkehrung deshalb auch als »Sättigungsformel $\aleph^{30}$ bezeichnet.

Deut 6,10-12

V. 10 Und wenn es geschieht, dass dich Jhwh, dein Gott, in das Land führt, das dir zu geben er deinen Vorfahren, Abraham, Isaak und Jakob, geschworen hat:

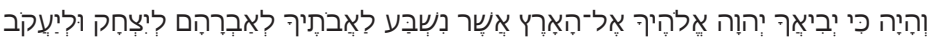

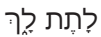

grosse und schöne Städte, die du nicht gebaut hast,

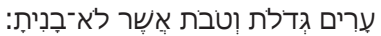

V. 11 Häuser, voll von jeglichem Gut, die nicht du gefüllt hast,

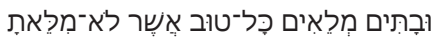

und ausgehauene Zisternen, die nicht du ausgehauen hast,

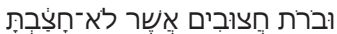

Weinberge und Olivengärten, die nicht du gepflanzt hast.

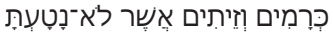

Und wenn du davon isst und satt wirst,

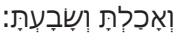

V. 12 dann hüte dich, dass du nicht Jhwh vergisst,

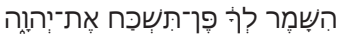

der dich herausgeführt hat aus dem Land Ägypten, aus einem Sklavenhaus.

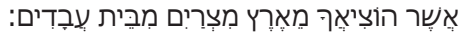

In Jes 65,21-23 findet sich eine Abwandlung dieses Versprechens. Der Text fokussiert auf der Stabilität der Ordnung, dass diejenigen,

\footnotetext{
${ }^{29}$ Die Wiederaufnahme des Weinbaus als Signal für den Anbruch der Heilszeit findet sich auch in 2 Kön 19,29; Hos 2,17; Jes 24,7-11.Vgl. ausführlich Hermann-Josef Stipp, Jeremia 25-52, Tübingen 2019, 240.

${ }^{30}$ So Podella, Notzeit-Mythologien und Nichtigkeitsfluch, 442-444.
} 
die etwas tun, auch davon profitieren werden. ${ }^{31}$ Die Handlung ist also nicht mehr vergeblich, sondern Tun und Ergehen entsprechen sich.

Jes $65,20-23$

V. 20 Dort wird es keinen Säugling mehr geben, der nur wenige Tage lebt,

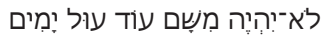

und keinen Greis, der seine Tage nicht vollendet,

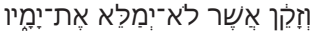
denn ein junger Mann wird sein, wer mit hundert Jahren stirbt,

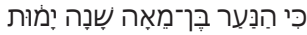
und wer hundert Jahre nicht erreicht, gilt als verflucht.

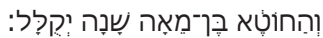

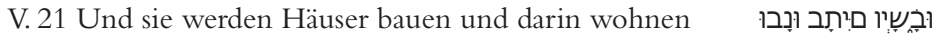
und Weinberge pflanzen und deren Früchte essen.

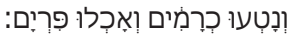

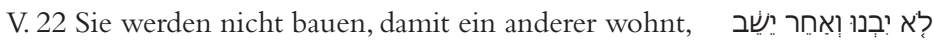

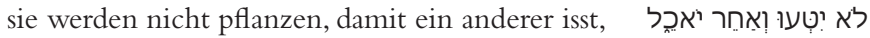
denn das Alter meines Volks wird sein wie das Alter des Baums,

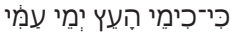

und was ihre Hände erarbeitet haben, werden meine Auserwählten geniessen.

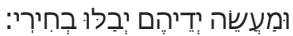

Dabei wird auch in der Heilprophetie, die Grenze des bereits Erfahrenen überschritten und die Zukunft utopisch: Kein Säugling wird mehr nur wenige Tage leben; alle Menschen werden mindestens hundert Jahre alt. Alle menschlichen Bedürfnisse werden befriedigt und eine gerechte Gesellschaftsordnung garantiert.

\section{Zwischen Unheil und Heil, Angst und Hoff- nung...}

Angst und Hoffnung werden in der Hebräischen Bibel mit sehr ähnlichen Bildern und unterVerwendung einer ähnlichen Terminologie ausgedrückt. Im Vordergrund stehen die natürlichen menschlichen Bedürfnisse, insbesondere Hunger beziehungsweise Sättigung, die jedoch sowohl in den Unheils- als auch in den Heilszusagen über

${ }^{31}$ Vgl. Konrad Schmid, Schriftgelehrte Traditionsliteratur. Fallstudien zur innerbiblischen Schriftauslegung im Alten Testament, Tübingen 2016, 187-188, hier 198, "Jes 65,17-25 möchte mit seinerVerheißung betonen, dass [...] in keiner Weise mehr die Möglichkeit bestehen wird, dass deren gute Ordnung pervertiert werden wird." 
jegliche konkrete Erfahrung hinausgehen können und eine bislang nie dagewesene negative beziehungsweise positive Zukunft schildern.

Vermutlich - dies ist zumindest die gängige Annahme - reagiert dabei die Heilsprophetie auf die ihr vorangehende Unheilsprophetie aus dem 8./7. Jahrhundert, die ihrerseits im Kontext aramäischer und neuassyrischer Vertragstexte steht. ${ }^{32}$ Im Vordergrund geht es um die Androhung des Nahrungsmittelmangels: Wer übermäßig konsumiert, wird Mangel erleiden. Dieser konkrete Tun-ErgehenZusammenhang wird später noch verschärft und durch eine grundsätzliche Vergeblichkeit ausgedrückt: Obwohl die Menschen essen, werden sie nicht satt werden. Es ist nicht anzunehmen, dass das hier angekündigte Unheil - sei es eine Dürre oder die Eroberung durch die Assyrer oder Babylonier - direkt im Blick ist. Während die Kritik sehr konkret formuliert wird und sich auf einen spezifischen historischen Kontext bezieht, bleibt die Strafandrohung merkwürdig offen und schillernd, was eine authentische Prophetie und nicht ein vaticinium ex eventu wahrscheinlich macht. ${ }^{33}$ Sicherlich wird hier jedoch nicht nachträglich eine Katastrophe erklärt und nach Ursachen gesucht. Genauso offen und unkonkret ist schließlich auch die Heilsprophetie formuliert, die zum Zeitpunkt ihrer Entstehung nicht eingetroffen ist (teilweise auf Grund ihres utopischen Charakters auch gar nicht eintreffen kann), sondern Menschen in äußerster Not verheißen wurde. Sie wird - anders als die Unheilsprophetie - nicht mit dem Handeln der Menschen verknüpft, sondern schöpft ihre Kraft aus dem Versprechen Gottes an sein Volk.

Vielleicht ist es gerade in der aktuellen Zeit besonders nötig, Dystopie und Utopie enger zusammenzudenken als dies je zuvor geschehen ist um auch die entgegengesetzten Emotionen besser zu vereinen. Dass Emotionen eine sehr wichtige Rolle spielen in der Kommunikation über den Klimawandel ist längst offensichtlich. ${ }^{34}$

\footnotetext{
${ }^{32}$ Vgl. ausführlich Ehud Ben Zvi, Utopias, Multiple Utopias, and Why Utopias at All? The Social Roles of Utopian Visions in Prophetic Books within Their Historical Context, in: Utopia and Dystopia in Prophetic Literature, hg. v. Ehud Ben Zvi, Helsinki/Göttingen 2006, 55-85.

${ }^{33}$ Vgl. Jeremy D. Smoak, Building Houses and Planting Vineyards: The Early InnerBiblical Discourse on an Ancient Israelite Wartime Curse, in: JBL 127 (2008), 19-35, hier 35, hält fest, dass »the relatively imprecise and vague nature of the curses imagery allowed it to be customized and reformulated for various historical and social circumstances."

${ }^{34}$ Sefat Salama/Khalil Aboukoura, The Role of Emotions in Climate Change Communication, in: Handbook of Climate Change Communication, Vol. 1, hg. v. Walter Leal Filho et. al., Cham 2018,137-150, hier 145:»There is an urgent need to consider
} 
Auf der einen Seite steht (exemplarisch) die Stimme der sechzehnjährigen Greta Thunberg. In nur einem einzigen Jahr hat sie

- am World Economic Forum in Davos (22. Januar 2019) den Mächtigsten der Welt ihre Tatenlosigkeit vorgeworfen,

- den SchauspielerInnen und Prominenten in ihrer Dankesrede bei der Auszeichnung mit dem "Special Prize Climate Protection" (Goldene Kamera, 5.April 2019) in Berlin vergehalten, dass sie sich gegen alle Ungerechtigkeiten auflehnen, aber die Klimagerechtigkeit missachten würden, weil es sie einschränken würde um die Welt zu fliegen in ihre Lieblingsrestaurants, an ihre Lieblingsstrände und Lieblingsyogaseminare

- und alle, die nach wie vor behaupten, alles würde gut werden, getadelt, dass sie nicht auf die Wissenschaft hörten (beispielsweise in ihrer Rede am 5. Dezember 2019 im House of Representatives, Washington, DC).

Ihre Botschaft richtet sich sehr gezielt an die jeweilige HörerInnenschaft, aber ihre Grundbotschaft ist überall die Gleiche. Sie droht und will aufrütteln: »I want you to panic! $\aleph^{35}$ Auf der anderen Seite steht (erneut exemplarisch) der Sozialpsychologe Harald Welzer, der mit einer neuen Gesellschaftsutopie beschwichtigt und verheißt, dass alles auch ganz anders sein (oder werden?) könnte, wenn das Utopische erst realisiert wird. ${ }^{36}$ Beide Perspektiven, Angst und Hoffnung, müssten sich vielleicht auch heute an ähnlichen Vorstellungen und Bildern orientieren, wenn daraus eine wirkliche Vision für die Zukunft werden soll.

- Dr. Sara Kipfer ist Margarete von Wrangell Fellow an der Universität Heidelberg. Zu ihren Forschungsschwerpunkten gehören neben der Rezeptionsgeschichte, die alttestamentliche Anthropologie (speziell Körperkonzepte und Emotionen) sowie Deutungsund Bewältigungsmuster von Katastrophen in der Hebräischen Bibel.

emotional aspects when we discuss the implications of climate change and how to communicate adaption and mitigation to climate change. To overcome the challenges and barriers to engagement with climate change, communicators must be critically aware of the role emotions have in prompting behavior, and should continue to develop an understanding of the ways in which people emotionally engage with climate change."

35 Vgl. https://www.fridaysforfuture.org/greta-speeches (04.01.2020) sowie Greta Thunberg, Ich will, dass ihr in Panik geratet! Meine Reden zum Klimaschutz, aus dem Englischen von Ulrike Bischoff, Frankfurt a.M. 2019.

36 Vgl. Harald Welzer, Alles könnte anders sein. Eine Gesellschaftsutopie für freie Menschen, Frankfurt a.M. ${ }^{6} 2019$. 